\title{
Mitiger les pratiques corporelles des cultures des migrants et les activités physiques et sportives scolaires
}

\section{Henri Boularand}

\section{CpenEdition}

Journals

Édition électronique

URL : http://journals.openedition.org/corpsetculture/865

DOI : $10.4000 /$ corpsetculture.865

ISSN : 1777-5337

Éditeur

Association Corps et Culture

Édition imprimée

Date de publication : 1 janvier 2004

ISSN : 1268-5631

Référence électronique

Henri Boularand, « Mitiger les pratiques corporelles des cultures des migrants et les activités physiques et sportives scolaires », Corps et culture [En ligne], Numéro 6/7 | 2004, mis en ligne le 11 octobre 2007, consulté le 08 septembre 2020. URL : http://journals.openedition.org/corpsetculture/ 865 ; DOI : https://doi.org/10.4000/corpsetculture.865

Ce document a été généré automatiquement le 8 septembre 2020

(c) tous droits réservés 


\title{
Mitiger les pratiques corporelles des cultures des migrants et les activités physiques et sportives scolaires
}

\author{
Henri Boularand
}

«Pour pouvoir mieux se délimiter il faut greffer
de l'ouvert. (...). L'ouvert ce n'est pas être
enfermé dans le dit, ce n'est pas seulement
parler ; c'est une disposition. (...). L'ouvert c'est la
possibilité de délimiter le singulier " .
Jean Oury ${ }^{1}$

1 Les raisons de tout groupe clandestin de se cacher sont nourries par un sentiment vécu d'injustice. Qu'il y ait manipulation ou non par ses chefs, les individus qui le constituent convergent vers le même but : y mettre fin, quitte à mettre leurs propres vies en jeu.

2 Le philosophe Gilles Deleuze et le "psychanalyste» Félix Guattari (1972), se sont attachés notamment à montrer que l'engagement pour la guerre est vécu comme une nécessité par ceux qui la font, dès lors qu'ils ont le sentiment que leurs conditions de vie leur sont devenues insupportables. En complément, les travaux de Norbert Elias sur la violence peuvent servir au plan psychologique, d'inventaires et d'analyses des raisons pour lesquelles des formes diverses et individuelles de ressentiment s'additionnent pour se rassembler, se densifier dans des groupes - comme les migrants - difficilement identifiables sociologiquement, avant de finir par exploser (Elias N., 1994) $)^{2}$.

3 Le terrorisme qui fait l'actualité d'aujourd'hui pose au fond la question du sens qui est donné à la culture, c'est-à-dire du sens de la vie et, relativement à elle, du sens des valeurs et des politiques qui peuvent en rendre compte. Il déstabilise les consciences des témoins lorsque ces derniers voient les auteurs périr avec leurs victimes. La vie ne vaudrait-elle pas pour eux la peine d'être vécue ? Pour ceux-là les valeurs se situent audelà de la vie biologique et sociale. Cette forme actée de ressentiment est probablement 
très fortement ressentie psychologiquement au fond, par ses auteurs, non seulement comme l'expression du sentiment " d'une existence digne devenue impossible » mais aussi comme une opportunité d'accès à une vie meilleure assurée dans l'au-delà. Curieux mélange d'une mise en perspective d'un bonheur extrême dans l'imaginaire et d'un refus d'une vie appréhendée à travers d'incessants malheurs.

Ce ressentiment prend du temps avant que la violence ne sourde et n'éclate. Il est d'abord vécu clandestinement dans l'intimité du sujet, qui ne trouve pas de mots pour le dire. S'il y a des formes extérieures de la violence qui s'expriment différemment selon leur lieu d'incidence géopolitique, elle a des fondements psychologiques communs : un sentiment ressenti d'être victime de pressions et de répressions qui étouffent. Certains y répondent résolument en se faisant exploser avec des bombes; d'autres par des mots et des gestes qui peuvent aussi aller jusqu'à la déflagration si la pression continue et si les murs se refusent à porter des oreilles.

Il ne faut donc pas faire d'amalgame entre, là, le terroriste décidé et, ici, les attitudes de certains enfants de migrants qui ne sont que désorientés, tiraillés entre deux cultures en même temps qu'ils subissent une double pression. L'une qui relève de la poussée hormonale de l'adolescence, l'autre des regards qu'ils subissent parce qu'il sont différents en couleur de peau et en patronyme par exemple. Il ne faut cependant pas sous-estimer les identifications possibles des auteurs de "petites » violences à ceux du terrorisme qu'ils peuvent prendre comme modèles.

6 C'est peut-être pour ces raisons que les premiers s'expriment, parfois, à travers des attitudes qu'il est aujourd'hui devenu urgent pour les éducateurs de savoir décoder à condition qu'ils soient "en veillance: c'est-à-dire être là, disponibles, prêts à accueillir " (Oury J., 2002). Sur leurs terrains ce sont des demandes codées auxquelles répondre dans le même registre que dans celui où elles s'expriment, c'est aller droit à la faute face à des traits de comportement inattendus ; regards à la fois vides, agressifs, torves et fuyants : " conversations » à travers des combinaisons de borborygmes, onomatopées, lapsus, injures ou félicitations - : "Ah enculé! T'as eu le bac!", acting out et, pour finir, passages à l'acte. Toutes ces expressions ne sont pas à prendre au mot, mais à la lettre codée. L'éducateur doit remonter tout le parcours fantasmatique des locuteurs et y identifier les symboles à reprendre, décousus çà et là. La demande se tapit derrière ces écrans de langage et d'attitudes courbes.

7 La progression est inexorable dès lors que s'accumulent, par petits engrangements successifs, les insultes de la vie, les arrogances et les silences dans lesquels sont tenus les individus qui vivent ces infortunes. Cela finit par faire ambiance, chacun prend « une gueule d'atmosphère" et le phénomène se massifie par contagion. Cela devient dangereux pour tous, y compris pour les nantis au plan matériel et les moins mal servis en bonheurs et satisfactions diverses: quand les plombs sautent chez certains tout le monde se trouve dans l'obscurité. Chacun est alors contraint de se mouvoir à l'aveugle au milieu d'un monde où la violence répond présente à l'appel des aléas de l'histoire et, par exemple, aux inepties des attitudes et des politiques éducatives décalées et obsolètes.

8 L'allusion aux événements violents « du? " «11 septembre » et qui ont beaucoup surpris aux Etats-Unis est maintenant identifiée par le lecteur. Avec un recul de quelques mois émerge à sa conscience cette violence entretenue comme un bruit de fond permanent par les médias au moyen des informations diffusées au quotidien. Mais il peut se demander en quoi cela entre dans le cadre d'une publication regroupant des 
contributions à une réflexion sur le Métissage des Cultures. Les propositions qui suivent répondront en partie à cette question.

9 L'attitude des enseignants à l'égard des élèves issus de l'immigration est, le plus souvent, significative d'une volonté irréprochable en soi de dispenser à tous des connaissances sur le même mode de référence aux valeurs républicaines. Cependant le souci légitime d'assurer une égalité des chances entre ces derniers s'exprime avec une telle force que les différences - du point de vue des origines culturelles - sont vécues, au nom même de l'égalité, davantage comme un obstacle que comme une opportunité extra ordinaire de poursuivre des objectifs d'égalité permettant à tous d'accéder au savoir de manière appropriée à chacun. Ainsi, ce qui est vécu comme barrière à l'égalité est « jugé » de facto comme étant négatif, sans que cela soit clairement formalisé. Cela ne peut que choquer les sensibilités des enfants de migrants et rendre difficile, relativement à leur cadre familial, la reconnaissance des fondements de l'autorité symbolique des parents qui s'expriment au quotidien sur les supports de leur culture d'origine propre. Peuvent s'ensuivre, en retour, des perturbations au niveau psychologique avec des comportements susceptibles d'aller jusqu'à des manifestations à violence plus ou moins exacerbées.

10 Pour contribuer à éviter de tels gâchis, peuvent être envisagées des reconnaissances en acte des cultures concernées prenant en compte de manière valorisante certains de leurs aspects positifs. Le professeur d'éducation physique est un acteur qui gagnerait à systématiser ce type de démarche en apportant sa pierre en matière de cohérence aux projets pédagogiques et d'établissement. Aussi, afin de poursuivre un tel objectif, lui est-il nécessaire de fournir l'effort de travailler son regard sur les aspects positifs des différences culturelles qu'il côtoie au quotidien de l'école en les reliant aux vastes enjeux historiques caractéristiques du monde d'aujourd'hui au plan du mixage des cultures.

Le métis, un navigateur « inter-autres » analyseur des transformations culturelles

11 Un remodelage des frontières s'opère dans le cours de l'histoire. Les no man's land qu'ils soient inter-étatiques ou psychologiques sont changeants, fluctuants, mobiles. Ils traversent les pays et les modes de pensées séquentielles des villes et des villages partout dans le monde, se transfèrent d'un continent géographique et de celui d'un mode de pensée à un autre, se mélangent avec une logique incontournable, aléatoirement, sans prévenir. "Ce qui a été ne sera plus»: les redistributions géographiques, géopolitiques, "géoculturelles » confondues du monde sont de plus en plus imprévisibles. Or, ce ne sont pas les organisations, les organismes internationaux qui sont porteurs de ces changements. Ce sont les individus tous liés au même destin comme cela n'a probablement jamais autant été le cas et d'une manière aussi significative que cela l'est aujourd'hui dans l'histoire des hommes. En effet, les stratégies des organisations et des organismes sont non seulement décalées mais encore s'élaborent le plus souvent, a contrario des dynamiques individuelles en marche (Salamé G.,1996) ${ }^{3}$.

12 Les migrants sont de ce point de vue des vecteurs significatifs des transformations culturelles en jeu. Le migrant, en situation dans un espace et une culture donnés, mérite d'être "ciblé » comme un porteur non négligeable de changements, à l'instar des artistes que Marshal Mac Lugan invite à appréhender comme les "radars de la société ». Si le radar efficace, à savoir l'artiste parfois maudit de son vivant, peut être porté au pinacle après que son œuvre se trouve installée objectivement dans le monde - 
dimensionnée, mesurable-, elle est exposée aux regards et suscite la préhension esthétique; le migrant quand il est maudit de son vivant, n'en laisse pas moins des traces de ses apports originaux. Sa reconnaissance positive est toutefois d'une immédiateté beaucoup plus rare que celle de l'artiste bien qu'ils soient tous deux engagés dans un même processus d'expression de certaines formes du monde distinctes de celles habituellement perçues dans les routines culturelles quotidiennes d'une société donnée. Selon Pascal, «figure porte absence et présence» : sa présence est très indirecte quand elle est celle du migrant à travers le couscous, le raï, par exemple, le regard porté sur ce dernier transite par les voies digestives et auditives en l'espèce. Ainsi donc, la cuisine, la musique, se présentent-t-elles à l'autochtone français par exemple comme des assimilations annonciatrices d'une reconnaissance qu'il consentira plus tard une fois que les éléments culturels importés auront été incorporés et engrangés dans la mémoire des goûts et dans celle des rythmes. Dès lors, les colporteurs d'éléments de cultures étrangères ne seront plus nécessaires. Mais cela prendra beaucoup de temps. (Boularand H., 2000).

13 Le regard négatif porté sur le migrant et le déni de sa culture d'origine en ajoute au temps. Ce mépris est tenace. Il a une histoire longue et a pris une dimension archétypique tellement ancrée dans les consciences qu'il continue d'opérer à travers des grilles d'affects péjoratives et exclusives dont l'éducation physique, le sport ont aussi été le théâtre (Boularand H., 1999) 4 .

14 Elle remonte à l'occupation coloniale des terres d'Afrique et d'Asie notamment. Celle-ci a été une opportunité de domination militaire, politique et culturelle ne laissant aucune place à une prise en compte favorable des cultures indigènes et qui fût occasion de s'en inspirer volontairement dans le but de s'en enrichir. Localement, le droit n'était pas le même pour les dominants et pour les dominés. Des usages locaux de police et de justice ne répondaient pas aux lois telles qu'elles étaient appliquées sur le territoire national français. Il en allait de même pour le droit à l'éducation (Léon A., 1991).

Dans ces conditions, une mise à distance de deux «types » de culture s'est installée et fixée dans les usages et dans les consciences. Elle se perpétue bien au-delà de la fin «officielle » de l'ère coloniale qui a duré plus de cinq siècles sur certains territoires (Coubertin P. s.d.) $)^{5}$.

16 Les émigrations depuis les anciennes colonies vers la métropole ont été de plus en plus importantes à partir des dates des indépendances nationales pour atteindre les proportions connues aujourd'hui. Les migrants qui en sont issus continuent d'être l'objet, au plan culturel, des mêmes mises à l'écart opérées dans leurs pays d'origines par les coloniaux. L'exclusion culturelle est encore très vivace dans l'éducation française institutionnalisée à l'égard des migrants d'aujourd'hui, comme elle l'a été autrefois à l'égard des cultures régionales françaises qui ont connu aussi leur période de mépris et d'expression interdite et sont encore l'objet d'une certaine indifférence (Marchal J-C.,1997) ${ }^{6}$. Si le migrant est sollicité, reconnu et retenu comme force productive, il est, institutionnellement nié en tant que porteur d'une " force culturelle " dont le potentiel est de facto indéniable. Il se voit donc contraint d'occuper un vide qui n'a pas le même sens pour tous.

17 "La nature a horreur du vide». Si cette vision pleine de justifications rationnelles continue de nourrir les représentations et la volonté des puissants de ne laisser aucun espace rentable économiquement livré à lui-même, le vide ne leur est pas totalement accessible pour autant. Il laisse toujours des espaces où les migrants dessinent des 
trajets d'expatriation. Ceux-ci en fonction des pressions et des dépressions politiques et économiques diverses traversent aléatoirement le monde en franchissant les mers, en s'installant ici et là. Ces vides-là, comme les vents, ne connaissent pas de frontières. Les migrants s'y engouffrent quels que soient les interdits qui veulent leur être opposés pour les empêcher de les franchir.

C'est dans ces mêmes lieux, sans dehors ni dedans stables et arrêtés conceptuellement, que s'exprime la culture en mouvement: elle n'a de cesse de s'immiscer dans ces espaces interstitiels inexplorés. Ainsi ces vacuoles sont-elles des enjeux de lutte entre, d'une part, la reproduction sous des formes de pouvoirs politiques et économiques renouvelées en permanence et très significatives d'une volonté de conserver ses privilèges à tout empire établi, et, d'autre part, l'expression des originalités, de la créativité et des innovations propres au versant dynamique de la culture occupé par le migrant par nécessité. Là se dessine une problématique forte, en localisant bien le migrant au pivot de cette alternative et en le considérant comme l'occupant d'un espace où du changement s'opère.

19 La culture, à travers ses manifestations, a pour fonction de révéler et de publier l'homme individuel nouveau qui s'annonce dans la multiplicité d'une histoire culturelle au pluriel et de s'ouvrir à tout lecteur déjà sensible à cette histoire. Celui-là même qui, après avoir froissé et trituré des représentations du monde qui lui sont devenues insupportables car colportées par une culture qui lui apparaît obsolète, se donne le change en modifiant subrepticement sa mentalité. C'est ce processus de combinaison qui donne une image globale, bien que floue et indécise, que quelque chose change dans la culture et les représentations du monde (Buci-Glucksmann C., 1998) ${ }^{7}$.

Cela ne se réalise pas sans que tous ceux qui se rencontrent ici: ceux qui viennent de loin comme ceux qui ont "toujours" été ici, soient confrontés - du fait même de l'addition des référents culturels que constitue cette rencontre - à un problème d'hypertrophie identitaire. C'est, par exemple ce que Lacan évoquait à travers le concept de "forclusion du nom du père » qui peut être traduit schématiquement ici par la situation dans laquelle se trouve tout candidat à la construction de son identité, autrement dit au transfert, lorsqu'il se trouve pris au milieu de deux référents symboliques.

21 Or ce ne sont pas des dogmes ou des idéologies politiques ou économiques qui peuvent décider d'organiser ce type de changement. Ce dernier qui ne cesse d'advenir vient plutôt des refus de ce qui existe déjà de la part de ceux qui font feu de tout bois pour affirmer la volonté de dépassement individuel (Beji H., 1997) ${ }^{8}$. Par exemple, sous l'apparente débilité des modes ou des mysticismes se cachent des enjeux identitaires forts qui concernent tout individu de quelque culture d'origine qu'il soit.

22 L'exemple suivant servira d'illustration. Sous les habits de la culture, se cache pudiquement un nombril que la modernité véhiculée à travers le monde appelle à exposer. Montrer son nombril, comme cela est devenu «la mode » depuis quelques années pour certaines femmes, en portant des hauts courts et des hauts de jupes ou de pantalons bas sur les hanches, ne se corrèle pas exclusivement avec volonté de séduction ou érotisme. Quelques uns s'y trompent : se croyant dans ces cas face à des séductrices animées d'un irrépressible désir sexuel, ils cherchent donc à répondre en montant des plans de séduction à caractère érogène. Ils s'y aventurent avec une imprudence qui peut les conduire à quelques revers. Au contraire, cette expression est plutôt à prendre au premier degré suivant: "Voici ce qui m'attache à la vie, moi en tant 
qu'individu, comme cela m'a attaché à ma mère. Toute la différence d'avec cette dyade originelle c'est que je prends désormais le monde à témoin que j'existe. C'est pour cela que je révèle mon intimité: mon nombril est ouvert et tendu vers le monde». A force de couper par étapes successives les cordons qui les séparent du vaste monde dirait Winnicott, pour faire définitivement la preuve du fort désir d'ouverture la béance ombilicale leur tient lieu de pièce à conviction : c'est l'absence obscène - donnée en spectacle - du cordon qui signe l'autonomie et la liberté de sa propriétaire. Ainsi va le changement dans le procès de mise à jour de l'individualité. Là, la mode sert de biais stratégique et tactique, plus ou moins préparé, pour affirmer une existence propre.

Le même objectif d'affirmation individuelle est probablement poursuivi par ces jeunes femmes très vêtues et portant le tchador. Manière de faire connaître au dehors leur détermination à rappeler cette trahison originelle à l'égard de Dieu au Jardin d'Eden et de dire qu'elles ont choisi de faire acte de contrition - le mot hébreu qui désigne le vêtement a la même racine que celui qui signifie trahison. Cette attitude, chez des femmes qui, au demeurant, tiennent des discours témoignant de l'intégrité de leur intelligence, de leur cohérence logique, de leur aplomb psychologique et de leur sagacité est très déstabilisante pour un occidental qui, dès lors, ne peut plus, sauf à donner un verdict injuste et débile, évoquer une quelconque "victimisation" sacrificielle les ayant conduites à se réprimer de l'intérieur - répression intériorisée, comme il a tendance à le dire avec le jugement sans appel de l'expert. Ces deux exemples montrent que sous des dehors culturels il s'agit probablement en fait de choix individuels d'attachement à la vie ; existentiels donc.

Face à la diversité de ces choix se présentent deux éléments d'alternative, le refus ou l'acceptation.

25 A l'évidence l'acceptation doit l'emporter au nom du respect des libertés individuelles et ce d'autant plus que la jeune fille voilée ne cherchera pas à couvrir de force le nombril dénudé de sa voisine sur les bancs de l'école par exemple, pas plus que la seconde n'use de violence pour arracher de force les vêtements de la première, chacune respectant les itinéraires identitaires de l'autre. Parce que, sans le savoir même, peutêtre font-elles en quelque sorte partie du même club où tacitement la finalité d'association tourne autour du désir d'affirmer l'identité de chacun de ses adhérents. Les enjeux sont les mêmes, bien que définissables à travers des codages différents. Dans ce contexte, le spectateur de ces appels sémiologiques multiples est, d'un point de vue phénoménologique, réceptacle de perceptions croisées : il est de facto un témoin métis d'un environnement d'autant plus mixte que se multiplient les télescopages culturels. Le spectateur pur et neutre n'existe dès lors que dans l'imaginaire. Contraint par la multiplicité des référents culturels portés dans un espace donné par différents représentants de cultures différentes, il ne peut pas faire autrement, sauf à se discréditer par son peu de capacité à percevoir un monde divers, que de prendre en compte la forme au moins elliptique - à deux centres donc - que prend la culture qui s'ouvre à lui. travers ces no man's land, espaces d'errance qui pourraient être identifiés comme des «lieux neufs» - un peu comme ce que les historiens appellent des ruptures - que se construisent à petits pas des bases pour les refuzniks de tout bord, en solitaire ou en groupes plus ou moins organisés. Ils redessinent, de manière improvisée et inédite, agressive, du lien social en excitant les dormeurs. Ces satisfaits des routines d'une 
harmonie faite, en musique, par exemple, pour le confort d'écoute d'une mélodie qui de manière convenue ne cherche surtout pas à étonner autrement que par la répétition plus ou moins bien exécutée de ce qui est déjà connu. Une violence qui heurte, quand elle ne tue pas, tant ses auteurs ont souffert de se trouver contraints dans l'ombre ils décompensent en se livrant au chaos pour que ça bouge.

En fait le métissage ne peut pas relever exclusivement du migrant migrateur d'un espace géographique à un autre. Par la conséquence même de son nomadisme, ce dernier porte en lui une structure où se cristallisent de facto les processus mêmes du métissage: il ajoute ainsi irréversiblement de la nouveauté au paysage culturel. Inévitablement il contribue d'autant plus fortement à transformer les «cadres culturels » habituels qu'il "vient de loin». Il est objet de transfert pour ceux qui "l'accueillent" non sans ambiguïté, c'est-à-dire à la fois avec des défenses et des attirances. Face à face, le migrant comme l'autochtone sont chacun un miroir où l'autre se projette en quête de sa propre humanité. A l'aventure du dépaysement culturel accompagnant nécessairement le déplacement géographique de l'un s'ajoute, dans un même espace et dans un même temps sociaux, celle d'une remise en question des limites culturelles de l'autre et obligeant celui-ci à migrer, aussi, vers de l'inédit. Ce type de rencontre remue les impétrants. Quand il fait peur il maintient les distances entre eux, quand il fascine il les rapprochent avec le plus souvent une forte ambivalence affective.

L'autre, entre crainte et fascination

Aujourd'hui l'heure est donc à la fusion des pratiques culturelles pour l'avènement d'un homme nouveau, nécessairement lié au sort de ses semblables pris dans des catastrophes que l'information télé-médiatique diffuse quasi instantanément et au quotidien à travers toute la planète. Par quelle bizarrerie l'étranger en arrive-t-il jusqu'à devenir un objet d'identification?

"Nous sommes tous des Juifs allemands", harangue Daniel Cohn-Bendit repris par le chœur des manifestants de mai 1968 en se retournant contre le fascisme tout en tournant le dos à la Sorbonne. "Nous sommes tous des Américains" a-t-on lu dans la presse et entendu dire à droite et à gauche - de-ci, de-là -, au lendemain du 11 septembre 2001, déclaration de solidarité avec les victimes de la veille et contre le terrorisme. "Nous sommes tous des Algériens ", c'est ce que montre, agitant un drapeau algérien, ce jeune Français traversant, le 6 octobre 2001, la pelouse du Stade de France à Saint-Denis en s'intercalant, à la $76^{\text {ème }}$ minute du match, entre "l'équipe de football d'Algérie qui joue à domicile » et l'équipe de France qui joue chez elle. Il y avait conquis un espace vide, opportun pour faire émerger à la surface et en pleine lumière d'un théâtre mille fois rêvé auparavant son « algérianité » dressée comme un phallus, simulacre de sa toute puissance vers le ciel du Stade de France. Le score, quatre à un ou un à quatre, au moment où il provoque l'interruption de la rencontre n'est pas ce qui comptait pour lui et ceux qui l'ont rejoint dans son exploit. A quand les «Nous sommes tous des Musulmans et des Chrétiens palestiniens, des Juifs israéliens » ou encore " Nous sommes tous des estropiés ou des cadavres afghans et irakiens"?

30 Un problème se pose là où les acteurs cités de ces déclarations sont en phase avec l'événement sur un mode tragique, les autres devant leur poste de télévision le reçoivent sur un mode pathétique. Même en direct ou en simultané existe chez ces derniers un décalage affectif par rapport aux premiers: il n'y a aucune commune 
mesure entre les affects qui relèvent respectivement de l'un et de l'autre de ces deux types de postures.

31 Ces identifications passagères et mutantes au gré des événements posent la question de la fin des remords. Le remords comme conséquence d'une faute collective, soit, à condition de ne plus recommencer la faute ailleurs, collectivement et individuellement. Or, malheureusement l'histoire montre que se succèdent sans interruption des thèmes de natures différentes qui bousculent les habitudes, déclenchent des attitudes d'exclusion produisant elles-mêmes de nouvelles victimes. De nouveaux sacrifices s'opèrent et les regrets s'installent plus tard, trop tard : quand le mal est fait. Dans ces conditions, qu'en est-il de l'identification?

Le repentir prend racine comme si les âmes des victimes avaient élu domicile chez les bourreaux qui se prennent de passion quasi amoureuse pour elles. Leurs mets, leurs rythmes musicaux et leurs chants sont alors appréciés, leurs croyances religieuses suscitent l'intérêt - les ventes du Coran ont explosé en France, notamment depuis le 11 septembre 2001: il semble que la "victimisation " d'aujourd'hui opère comme la constitution d'un capital de culpabilité et de ressentiment pour demain.

Par exemple, la passivité entachée de collaboration à la Shoah de tristes acteurs d'hier a donné en legs à la génération d'aujourd'hui un bien curieux héritage. Les héritiers semblent ne pas toujours comprendre que c'est un enchevêtrement de causes multiples, jusqu'aux plus ténues en apparence, qui a conduit les générations qui les ont précédé à cette Catastrophe humanitaire. C'est pourquoi, en fait, ils en perpétuent ce qu'elle a de fonds commun avec toutes les formes de xénophobie, de racisme, d'exclusion, d'arrogance, en se cantonnant eux-mêmes dans une passivité détestable et en cautionnant au quotidien l'expression de toutes ces espèces d'injustice. Simplement, les victimes ne sont plus les mêmes et les méthodes ont changé de nature, bien que les résultats tendent vers les mêmes insultes à la dignité. Il ne suffit donc pas, examinant l'histoire, de se contenter de faire amende honorable en mettant toute son énergie au service du devoir de mémoire, encore faut-il, passé le cap de la culpabilité, s'engager dans un authentique processus de rédemption pour se défaire des violences cautionnées au quotidien dans l'indifférence et le repli frileux sur soi (Solemne M., 1998)'.

Cet itinéraire peut d'autant mieux être suivi que le migrant est considéré par l'hôte comme occupant dans son expérience et ses espaces quotidiens une position culturelle métisse qui l'incite à le rejoindre volontairement dans cette situation. C'est un moyen approprié de le reconnaître favorablement dans sa duplicité sans occulter les problèmes identitaires qu'elle génère en même temps qu'elle invite à prendre aussi une "position culturelle» double dans un processus de métissage inversé. Des intérêts mutuels réfléchis prennent corps dans la construction d'une culture double. Les voisinages avec les migrants peuvent ainsi évoluer du stade d'une cohabitation des cultures dans la juxtaposition à celui d'une liaison sociale dans le cadre dynamique et mixte d'une communauté des différences. Cette attitude responsable au présent engage l'avenir et dessine des perspectives optimistes.

C'est sur ce fond d'analyse que s'appuient les propositions de travail et de recherche pédagogiques en éducation physique évoquées. Elles visent la mise en œuvre d'une " utopie concrète » donnant corps au «Principe Espérance » chère à Ernst Bloch (Bloch E., 2001). Elles s'appuient sur une conception du rapport aux techniques d'enseignement et aux techniques corporelles engagées d'une manière qui ne déroge pas au "Principe Responsabilité » mis en exergue dans les travaux de Hans Jonas (Jonas H., 2001) et dont il 
est fait écho à propos des rapports qu'entretiennent aujourd'hui les techniques avec la biologie et l'éthique (Lecourt D., 2002). Les réflexions et problématiques développées par les trois derniers auteurs cités n'engagent pas moins les acteurs du champ de l'éducation physique institutionnalisée.

Au même titre que tout enseignant est lié au monde par l'actualité sociale et politique, bien au-delà des sphères des institutions éducative, familiale et domestique, il gagne à s'intéresser aux modes de pensée écologique et d'analyse systémique qui relient le macrocosme politique, scientifique à celui de l'engagement individuel aussi « mineur " qu'il y paraît à première vue. Les méthodes d'intervention en éducation physique peuvent tout à fait alimenter cette logique à travers les spécificités professionnelles des enseignants. Au demeurant l'ouverture au monde fait partie du métier de tout éducateur responsable.

Pour une approche ouverte du métissage

Les affects que soulèvent les événements brutaux, dramatiques et spectaculaires au niveau de l'opinion et à la surface des consciences individuelles ne sont que des signes extérieurs ponctuels relatifs à ce type d'expression brutale. A chaud les témoins y entrent en fusion. Le drame, un instant, ne se joue plus derrière le rideau fermé d'un théâtre. Si ce dernier reste toujours " en quête d'auteurs ", l'espace d'un instant, sa scène déborde d'acteurs qui prennent les choses en mains fortuitement, de manière inédite et le plus souvent désordonnée.

L'excitation, la colère, le désarroi, la peur provoqués par la rudesse d'un événement dramatique récent dans des pré-carrés anglo-saxons - occidentaux, et qui s'est déjà éloigné dans le temps - même si ses causes perdurent tant il est fait obstacle officiellement à leur identification et par conséquent aux moyens d'y mettre un terme au fond, il est alors temps de le situer, c'est-à-dire de tenter d'en faire l'étiologie. Il ne s'agit de rien d'autre que de répondre à une question : "Pourquoi est-ce arrivé?». Elle commence à se poser une fois passée l'étape productrice d'émotion qui accompagne les faits concernés. Chercher des réponses à cette question c'est se préparer pour l'avenir afin de n'être jamais surpris quand l'exclusion et la discorde semées, la récolte, en retour, inévitablement, ne peut que se solder par des catastrophes et la terreur. Il y a urgence, idéalement au moins, à tenter au plus tôt d'apporter sa contribution à son effacement.

Cela se joue au quotidien dans ses rapports à l'autre. Et pour les éducateurs que sont censés être les enseignants il s'agit d'une prise de position nécessaire, obligatoire eu égard à une conscience morale qui doit nécessairement les pousser à une lecture pertinente de ce qui se joue sur la planète métisse ici et maintenant à savoir une tragédie qui les entraînent eux aussi.

Dans ce processus d'approche et d'analyse des causes, doivent intervenir le bon sens, la raison et la prudence caractéristiques de toute approche épistémologique de la combinatoire des faits susceptibles d'avoir engendré l'événement. Il est utile d'explorer tous les champs d'expérience où il prend racine et de révéler des faits qu'une approche préalable de ces mêmes expériences aurait ignorés, occultés, considérés comme minimes et, pour tout dire, tenus pour négligeables. L'exclusion prend racine au quotidien et dans l'action individuelle. Dans les pays démocratiques, si elle ne se niche plus textuellement dans les lignes des constitutions républicaines par exemple, elle n'en existe pas moins avec une insistance déconcertante dans les usages sociaux. C'est là qu'il faut la débusquer car elle y insiste sans répit. 
41 Les problématiques des métissages portent à l'évidence à des interrogations multiples et à un processus de conceptualisation qui reste extrêmement ouvert, où les tâtonnements s'imposent au contraire de significations rationnelles bien arrêtées et closes à l'intérieur de critères de civilisation et de culture qui restent définitivement fixés dans certains imaginaires. Elles reposent nécessairement sur des configurations extrêmement floues qui appellent de ce fait l'acuité du regard et la quête du détail avec un œil acéré. En effet, les phénomènes de métissage sont par définition ancrés dans l'aléatoire, l'imprévisible donc. Vouloir les expliquer contraint les chercheurs à courir après en vain jusqu'à en perdre leur souffle. Le savant, comme pour les associations d'idées dont les captations relèvent plus de la psychanalyse que de quelque science que ce soit, est réduit à se contenter d'en mesurer les effets plus que d'en prévoir les manifestations nouvelles.

Choc des civilisations, tamponnages culturels, redistribution nécessaire des repères de la socialisation sont autant de faits sociaux évidents devant lesquels il faut cesser de faire l'autruche. Si «l'événement est un fait» selon Paul Veyne, le fait est multiple dans ses significations. Il est imprévisible, produit au gré de ces vents de mélanges dans les sociétés occidentales - "judéo-chrétiennes?». Il sur-prend, façon de dire qu'il arrache par le haut, comme s'il tombait du ciel, sauf pour le métis - mitigeur par nécessité s'il veut raison et vie garder, qui en a vécu la "préparation " au quotidien, à l'intime et terre à terre. Plus précisément, et ce avec des émotions fortes, il a vécu, comme un «agent double», le rejet de sa personne, contraint de ne parler que dans un camp culturel à la fois et de se taire dans l'un pendant qu'il parle dans l'autre et inversement. Situation éreintante qui le contraint, à chaque fois, à changer de conduite. « Familiarisé » à l'arrogance et aux insultes des porteurs des cultures dominantes, il est de facto un témoin atypique "averti »- non sur-pris donc, des manières excessives et explosives avec lesquelles il y est répondu par des semblables plus vindicatifs qui passent à la révolte lorsqu'ils ne peuvent plus endurer leurs souffrances (Franchini P., 1993 ; Fanon F., 1974) ${ }^{10}$.

Lancer des réflexions sur le métissage - des cultures, des modes de pensée, des corps et de leur déclinaison de couleurs - force nécessairement à naviguer entre les sciences pour y trouver des outils d'investigation. Bien que cette navigation soit incontournable quand il s'agit de préoccupations portant sur un tel thème, il est très difficile de dépasser le stade de l'émergence de bribes liées les unes aux autres sur un mode associationniste et qui se redouble de l'addition d'outils d'investigation tirés des disciplines scientifiques à travers lesquelles sont menées les recherches.

Pour en sortir, idéalement, a priori seule une approche encyclopédique est en mesure de rendre compte de ce qui, idéalement aussi, pourrait être considéré comme une manière complète pour aborder les questions posées par les phénomènes de métissage. Mais cela crée un problème : l'encyclopédisme a fait son temps à cause de la multiplication inouïe des savoirs à en juger l'étendue de l'inventaire des disciplines scientifiques et aussi parce que le monde est devenu tellement compliqué qu'il ne peut se satisfaire d'une vision globalisante, jacobine et centralisatrice portée sur ses réalités diverses et changeantes.

Dans ces conditions, si les encouragements à donner aux "bons métissages » peuvent être renforcés dans leurs motifs par des approches diagnostiques utilisant toutes sortes d'outils scientifiques, les remèdes et les pronostics ne peuvent ressortir que du bon sens commun et de l'adhésion de tous à la prise en compte des bénéfices potentiels des 
métissages dans le procès d'universalisation tout en sachant à l'avance qu'il n'y en a que dans le respect et l'entretien des différences. Celui-ci ne peut se dérouler qu'en même temps que les modèles des valeurs s'élaborent. Il y va plus d'un état d'esprit que d'une culture savante spécialisée et close à l'intérieur d'une discipline scientifique. En somme une éthique est nécessaire qui se fonde sur l'observance des droits de l'homme ouvrant voix au chapitre, aux êtres humains de toutes conditions confondues.

Un exemple des limites de la science à partir de la recherche en biologie porte à rire. Une étude a montré que les femmes, inconsciemment, sont d'autant plus attirées sexuellement (amoureusement?) que les partenaires «recherchés » présentent les caractères génétiques les plus éloignés de ceux qu'elles portent. Alors l'intérêt des Blancs pour la musique des Gnaouas ne serait qu'une étape intermédiaire vers la satisfaction d'une irrépressible fascination sexuelle pour leurs opposés en couleur?

Cela reste à démontrer car, en la matière, il ne faut pas se précipiter trop vite dans des analogies. Autrement dit la valse ou le tango ne suffisent probablement pas à séduire une femme Masaï. Attirée par son « contraire » biologique ? Soit, à condition peut-être qu'il ait ramené au campement la peau d'un lion tué lors de son initiation. Qui dit qu'elle ne préfèrera pas ce berger-guerrier à un lauréat de la coupe du monde de football, Blanc ou Noir? Pour la séduire le prétendant mettra-t-il d'autant plus de chances de son côté s'il est un Blanc, qu'il a tué un lion à la sortie de son adolescence et qu'il danse le tango et la valse dans un style on ne peut plus académique?

Éducation physique, intégration et métissage culturel

Ce regard amusé suivant l'évocation des événements terrifiants massés dans une actualité récente, et qui, à l'évidence, provoquent la stupeur de tous, peut faire question pour certains lecteurs qui pourraient taxer l'auteur d'immoralisme et d'impudeur à l'égard des victimes. Il ne mérite pas cette sentence car il n'a jamais manqué de "compassion" pour les victimes des injustices comme le montre bien ses précédents propos; ni avant «le 11 septembre » de New York, ni après cette date, en ce même lieu comme ailleurs. Ce "qui vient de se passer " ne l'étonne pas: il a cet « avantage » d'avoir toujours vécu physiquement et mentalement en témoin éveillé des provocations qui ont précédé ça et là dans l'espace et dans le temps ce type de réaction (Boularand H., 1999). Au demeurant, l'actualité fraîche des " petits conflits » rapportée par des médias qui assimilent maintenant le terrorisme à la guerre augure du pire à venir.

49 La pratique sportive de compétition, le Professorat d'Education Physique et Sportive qui caractérisent sa vie professionnelle sont des lieux, des institutions dont les types de pratiques et les idéologies d'exclusion qui les caractérisent trop souvent - par exemple, à cause de l'interprétation courte de la notion d'intégration qu'elles expriment - ne doivent pas être considérés comme étrangers aux quelques causes abordées dans le développement qui précède. Ils sont à prendre en compte dans toute approche étiologique qui s'attache à mieux comprendre pourquoi dans un pays à forte tradition d'accueil des migrants, un nombre malheureusement significatif de leurs enfants, voire de leurs petits enfants ont perdu leurs repères au point d'en devenir un phénomène de masse suffisamment important pour que ces derniers soient ressentis comme semeurs de trouble et une menace pour la tranquillité de tout un chacun.

50 Par ailleurs, leur présence - "sur le sol français » gagnerait à être appréhendée par "leurs hôtes", au contraire de la représentation précédente que les descendants immédiats des migrants inspirent. En effet, c'est plutôt une opportunité d'introduire 
une logique tous azimuts d'accueil et de reconnaissance individuelle de l'élève enfant de migrant, en situation scolaire, par exemple. En envisageant systématiquement des prises en compte programmées d'activités corporelles et de jeux issus des cultures d'origine de leurs parents et grands-parents. Il y a là des opportunités encore trop souvent insoupçonnées d'ouvrir le champ des pratiques à des habiletés et aux sensations et sensibilités qui les accompagnent. Outre les bénéfices identitaires par le recours franc et contractuel à la quête des origines culturelles à travers leurs jeux dans les relations pédagogique et éducative, elles peuvent avoir un double effet positif. Premièrement, enrichir les habiletés de tous les élèves; deuxièmement, dans une logique d'échanges, en rendre directement responsables les Français originaires «des cultures d'outre-mer", autant que ceux descendant des régions d'Europe et de France par exemple (Béart Ch., 1960 ; Blanchard J-C., 1990, 1997 ; Bui-Xuân G., 1995 ; Vichet M., 2000) ${ }^{11}$.

51 C'est à ces concessions que doivent se résoudre les Professeurs d'Education Physique et Sportive pour répondre aux finalités citoyennes de l'éducation où la volonté réitérée du législateur pointe, avec une insistance légitime, l'importance de placer l'élève au centre des préoccupations dans les projets et les programmes éducatifs et d'enseignement.

L'égalité des droits à l'éducation, ne signifie pas l'uniformisation des élèves à partir d'une référence culturelle nationale ou relevant d'une «civilisation» donnée et exclusive. La culture française n'est pas le centre du monde. La connaissance des droits de l'homme chez les enseignants passe par une connaissance des droits culturels et identitaires des élèves. La construction d'une identité psychologique passe inévitablement par la question des origines. Elles sont remontées historiquement à travers les bribes archéologiques de la culture des parents migrants, au hasard. Un grand érudit russe et juif - une forme de métissage comme une autre -, qui n'aura jamais connu la Palestine, déclarait à la fin de sa vie: «J'aspire à connaître le ciel qui a inspiré mes ancêtres ». Il n'y a aucun doute à avoir sur ce désir qui a dû animer toute sa vie. Dans ce cheminement les intéressés sont souvent seuls. La conséquence en est que l'imaginaire pris pour réalité, ils passent à côté de ce qui permettrait de fixer un repère symbolique d'identité. Au demeurant cela ne peut donner qu'une identité "fausse", incertaine. C'est comme si cela fonctionnait au regard de leur culture et de leur histoire sur le même procès que la démarche imaginaire de l'enfant dans sa tentative de reconstitution de la fameuse "scène primitive " montrée par Freud dans ses analyses de "L'homme aux loups ". Le problème c'est qu'à la différence de cette scène, étant isolés, esseulés, dans cette aventure ils confondent le registre de leur histoire avec celui de leur imaginaire et, dans ces conditions tout « rattrapage » symbolique devient très difficile.

53 C'est, pour l'éducateur donc, être capable notamment d'identifier que les accès au savoir sont très souvent empêchés par des crises d'identité graves chez les enfants de migrants qui s'ajoutent, à un moment donné de leur scolarité et de leur adolescence, aux incontournables crises d'acné et de poussée hormonale. Aussi ne faut-il pas s'étonner de l'explosion des conduites. Dans ces moments-là l'enseignant comme l'élève est au cœur des problèmes générés par une conception de l'intégration qui refuse en fait le métissage.

54 Certes ce mixage culturel est en mesure d'aboutir, sur le plan quantitatif, à un enrichissement technique en étendant des habiletés propres à la culture occidentale à des dimensions exotiques s'y surajoutant. Mais cela ne représente là que le gain 
comportemental des enjeux du métissage culturel rapporté aux activités physiques. Le plus important dans ce pari du mixage, ce sont les effets qualitatifs possibles qui peuvent en découler. Il présente une opportunité d'ouvrir au corps des perspectives qui, dans la quête officialisée des origines, transcendent les seules techniques sportives. Les menus sportifs sont donnés actuellement comme quasi unique issue de construction du corps au plan "ludico-physique ». L'idée de cette entrée en immanence d'un élève-sujet, bouleverse à l'évidence les schémas pédagogiques sécuritaires où l'enseignant se croit, par devoir, obligé de fournir à l'élève des produits tout faits, sans origine avérée : "Voici; servez-vous et prenez de ce qui est déjà là ». Il est en effet certain que cette quête en acte de la connaissance de ses origines, à travers l'identification des jeux d'origine, n'est pas facile. Il faut pour cela parcourir des itinéraires jonchés d'obstacles et d'incidents identitaires... mes origines m'appellent : je veux savoir... le présent me tient trop fort : je ne veux pas savoir. Il y a un ballottage éprouvant.

Certes, à gros travail pour l'élève, gros travail pour l'institution et les enseignants. Pour inviter ces derniers à s'y engager, le législateur ouvre une voie d'élargissement des représentations des enseignants en charge de l'éducation physique et sportive en proposant quelques orientations dans les programmes nationaux. Cette base officielle est une opportunité à saisir par les équipes d'enseignants et de formateurs soucieux d'enrichir le concept d'intégration en lui donnant des significations plus appropriées aux réalités d'aujourd'hui ${ }^{12}$.

Conclusion

«Il vaut mieux allumer une bougie que maudire l'obscurité ». Proverbe chinois

Il est important de noter, à travers un exemple particulier que l'application de telles propositions n'est pas près d'être gagnée. Une conférence, organisée par l'auteur dans le cadre des conférences des Instituts Universitaires de Formation des Maîtres valant pour la préparation au concours du Certificat d'Aptitude au Professorat d'Education Physique et Sportive a déplacé deux étudiants de licence qui venaient en intrus disaient-ils en demandant de les excuser (Boularand H., 2000). Aucun candidat au CAPEPS inscrit administrativement à l'Institut Universitaire de Formation des Maîtres concerné ne s'était dérangé. Certains parmi eux sont aujourd'hui Professeurs d'éducation physique et sportive. Les formateurs n'étaient pas plus présents. Etait invité à participer aux débats, Nicolas Bancel, spécialiste de l'histoire des migrations et alors Président de l'ACHAC (Association Connaissance et Histoire de l'Afrique Contemporaine), membre du C.R.C.S. du département STAPS de l'Université Paris XI Orsay, (Bancel N., Blanchard P., 1998). Il serait donc vain de penser faire de la clientèle sur ces thèmes!

57 En arriver à cette conclusion c'est sans doute faire le constat fataliste qu'il y a chez les enseignants des conceptions de l'intégration qui résistent aux faits et profondément enracinées : celles qui reposent sur une idée de la Nation refermée sur ses frontières et des valeurs culturelles qui lui seraient propres, arrêtées à des contours bien définis. Voilà une représentation qui fait injure à la réalité environnante. François $1^{\mathrm{er}}$, en son temps, en était sorti. La Renaissance qu'il a lancée a eu les effets que l'on sait. Y penser devrait quelque peu battre en brèche les fibres nationalistes et encourager à s'ouvrir.

58 Sur les questions abordées ici par exemple, chacun est en droit d'attendre que les « experts de l'éducation » du grand siècle de la mondialisation s'engagent, en résistant à 
toute tentation centralisatrice jacobine, à mettre en œuvre une Renaissance devenue nécessaire à l'évidence des conflits qui ont pris place autant dans les crânes des élèves notamment que dans l'école et ses abords plus ou moins immédiats.

Cela ne peut qu'incliner à continuer d'essayer de convaincre qu'il faut en changer. Le changement est à ce prix: quand seront saisies les opportunités qu'offrent les postures et les pratiques de métissage, dans la continuité des prolongements des routes ouvertes des épices et de la soie.

60 A cet égard, certes, l'avenir et les hasards feront loi. Il appartient cependant aux éducateurs, aux enseignants - y compris les universitaires - d'appréhender avec une acuité soutenue ces phénomènes migratoires irréversibles, appelés à grossir de plus en plus au cours des années à venir, et de les prendre en charge à la hauteur de leurs compétences avec l'intelligence et le bon sens qu'il se doit.

61 Cela peut commencer pour eux en faisant des exercices d'assimilation et de digestion de l'expression des diversités que leur présentent leurs élèves au quotidien de manière plus ou moins biaisée. Mais l'atteinte d'un but ne suit pas forcément des voies linéaires rectilignes et univoques : s'il y a des obstacles pour les élèves il doit bien y en avoir aussi pour les professeurs. L'affrontement et le rapport de forces des cultures n'est pas le paradigme approprié pour en sortir. A l'évidence des faits qui se répètent négativement et de manière compulsive les solutions sont ailleurs ${ }^{13}$.

\section{BIBLIOGRAPHIE}

Bancel N., Blanchard P. (1998) De l'indigène à l'immigré, Paris, Gallimard.

Béji H. (1997) L'Imposture culturelle, Paris, Stock.

Béart Ch. (1960) Recherche des éléments d'une sociologie des peuples africains à partir de leurs jeux, Paris, Présence Africaine.

Bloch E. (2001) Le Principe Espérance, Paris, Gallimard, T. 1 1976, T. 2 1982, T.3, 1991.

Boularand H. (1997) La rencontre culturelle avec les migrants vue à travers l'aventure coloniale. Proposition pour une forme de partage à l'école, in Bui-Xuân G., De Léséleuc E., Gleyse J., Comment peut-on enseigner une culture corporelle ? Tréma spécial, hors-série $n^{\circ} 1$, version CD Rom, Montpellier.

Boularand H. (1999) Passé colonial et présent de l'immigration : contribution à un nouveau regard sur l'éducation physique, in Gleyse J. et coll. L'Education physique au XXe siècle. Approches historique et culturelle, Paris, Vigot, coll. Sport + Enseignement, 141-158.

Boularand H (2000) Passé colonial et présent de l'immigration : contribution à un nouveau regard sur l'éducation physique, in Boyer P., dir., Bulletin de liaison et d'informations scientifiques de l'AFRAPS, 18 -nouvelle série - avril, 31-33.

Buci-Glucksmann C. (1996) L'CEil cartographique de l'art, Paris, Galilée. 
Bui-Xuân G. (1995), Jeu et sport: pour une réhabilitation des significations originelles. Le cas du Damnyé, in Entre tradition et modernité: le sport. Actes du colloque international: sport, culture, tradition, Agde, éd. Jeux méditerranéens, 49-57.

Deleuze G. et Guattari F. (1972) L’Anti-CEdipe. Capitalisme et schizophrénie, Paris, Ed. de Minuit. Coubertin P. (de) (s.d., prob.1930), Cinq siècles et demi d'activité coloniale, Ed. du Centenaire (1830-1930), extrait de Notre France.

Elias N. et Dunning E. (1994) Sport et civilisation, la violence maîtrisée, Paris, Fayard, 1ère éd. Quest of Excitement, Sport and Leisure in the Civilisation Process, Basil Blackwell Ltd, 1986.

Fanon F. (1968) Les Damnés de la terre, Paris, François Maspéro, $1^{\text {ère }}$ édition : 1961, avec une préface de Jean-Paul Sartre.

Franchini P. (1993) Métis, Paris, Ed. Jacques Bertoin.

Jonas H. (2001) Le Principe Responsabilité - Une éthique pour la civilisation technologique, Paris, Flammarion.

Lecourt D. (2002) Le principe de précaution, in Science et Avenir, mars-avril.

Léon A. (1991) Colonisation, enseignement et éducation, Paris, L'Harmattan.

Marchal J-C. (1997) Nécessité et paradoxe d'un traitement didactique des jeux traditionnels, in Bui-Xuân G., De Léséleuc E., Gleyse J., Comment peut-on enseigner une culture corporelle ? Tréma spécial, hors-série $n^{\circ} 1$, version CD Rom, Montpellier.

Oury J. (2002) France Culture, A voix nue, $1^{\mathrm{er}}$ février.

Salamé G. (1996) Appels d'empires, Paris, Fayard.

Solemne M. (1998), Innocente culpabilité, Dialogues avec Ricœur P., Rougier S., Le Loup J.-Y., Naquet P., Paris, Ed. Dervy.

Vichet M.. (2000) Tori et Uke : pour une éducation à l' « altérité subtile », Corps \& Culture, («Corps et Educations »), 5, 185-194.

Winnicott D.W. (1969) Objet et Phénomènes transitionnels, in De la Pédiatrie à la Psychanalyse, Paris, Payot.

Winnicott D.W. (1971) Le corps et le self, in Nouvelle Revue de Psychanalyse, 3.

\section{NOTES}

1. Jean Oury invité de l'émission «A voix nue », France Culture, $1^{\text {er }}$ février 2002. Né en 1922, frère du pédagogue Fernand Oury (co-auteur avec Aïda Vasquez de Vers une Pédagogie institutionnelle, Paris, Maspéro, 1968), il est psychanalyste, psychiatre, fondateur et directeur de la clinique psychiatrique de La Borde. Il a été en charge dans les années 1970 du groupe « Psychanalyse et Institutions » à l'Ecole Freudienne, du vivant de Jacques Lacan, et il anime actuellement à Paris un séminaire à l'Hôpital Sainte Anne sur "L'Angoisse ».

2. La « méthode » utilisée à travers le concept de configuration reste valablement applicable aux questions de l'immigration en rapport avec l'histoire coloniale qui toutes deux ont connu des configurations à violence exacerbées, sans pour autant que les partis qui s'opposent encore aujourd'hui relativement à l'immigration, en France notamment, en soient sortis : 
"Les cycles de violence constituent des configurations formées par deux groupes ou plus: par un processus de double contrainte, ces groupes s'enferment dans une peur et un méfiance réciproques, (...) ces configurations sont caractérisées par des explosions de violence particulièrement virulentes qui conduisent à la victoire de l'un ou de l'autre groupe, mais qui parfois aussi aboutissent à l'affaiblissement de tous les participants... » p. 34. La « méthode » foucaldienne proposant de lancer un processus de sensibilisation à un niveau social par la mise en exergue des intérêts des particuliers cantonnés à des "reliquats» (" laissés pour compte», en marge, non reconnus en tant qu'identité propre mais porteurs de valeurs à dimension universelle - $c f$. les travaux et les engagements de Foucault sur la folie, l'éducation, les homosexuels, les prisonniers, toute communauté et individus exclus et figés dans l'ombre des regards politiques, sociaux) croise ici le mode de lecture façon Norbert Elias pour compenser les difficultés d'identification des groupes en conflit. En effet, Norbert Elias part d'une distinction nettement marquée entre Whigs et Tories. Il n'en va pas de même quand il s'agit de migrants croisant des statuts de citoyenneté - française et/ou autre, de religion, de rang social. Dans ce cas-ci, les bornes marquant les postures qui s'opposent sont floues, évanescentes et requièrent des analyses à la fois plus fines et plus marquées d'incertitudes et d'affects et où les outils scientifiques sont à construire. L'observateur-analyseur est dès lors contraint de laisser place, inévitablement, aux tâtonnements notionnels avant toute phase de conceptualisation et de mise en œuvre éventuelle de processus éducatifs, par exemple, se donnant pour objectif la recherche d'un apaisement des conflits individuels et collectifs.

3. Ghassan Salamé se penche sur la volonté de certaines puissances étatiques de rétablir de véritables empires coloniaux.

4. Dans cet article, l'auteur présentait un certain nombre de "pièces à conviction " produites par une approche de l'histoire coloniale qui a scellé avec un ciment d'une résistance rare l'ostracisme raciste dont les effets se prolongent aujourd'hui à travers nombre d'attitudes hostiles aux migrants. Il s'agissait, comme ici pour le présent de l'immigration, d'approcher quelques apparitions manifestes de ce phénomène à certaines époques de l'histoire coloniale de la France dans le domaine de l'éducation physique et du sport.

5. Il s'agit bien du baron de Coubertin, l'inventeur des Jeux Olympiques modernes. Il produisit ce texte de datation quasi exhaustive de toutes les conquêtes coloniales de la France, à l'occasion des célébrations du centenaire de l'Algérie française en 1930. Il en retrace les grandes lignes. Coubertin ne laisse aucun doute sur ses affinités pour l'impérialisme colonial.

6. Jean-Claude Marchal pointe l'absence des jeux traditionnels régionaux français dans les programmes des collèges bien que « les études ethnologiques et sociologiques montrent que le patrimoine ludique est un élément constitutif indéniable de l'expression des identités locales donc des cultures ».

7. « Pour ne reprendre que la célèbre carte de Hereford (1300) carte de référence pour tout l'occident médiéval, le monde est un cercle avec Jérusalem, la céleste et la terrestre, au centre ». Catherine Buci-Glucksmann qui peut être qualifiée d'historienne de l'oeil, ouvre les yeux sur les transformations, dans l'art, des angles d'incidence de nos regards sur le monde, op. cit.

8. Intellectuelle d'origine tunisienne vivant en France Héji Béji affirme sa volonté de défendre liberté et initiative individuelles dans les processus d'accomplissement 
culturel : il ne s'agit pas de construire une identité sur des valeurs subies de sa culture d'origine contre les valeurs culturelles du pays d'immigration.

9. Dans Solemne M. (1998), op. cit., Paul Ricoeur «Le sentiment de culpabilité : sagesse ou névrose ", pp. 9-29, montre la nécessité de la culpabilité liée à la mémoire et au devoir d'éthique.

Il est important d'ajouter que si ce devoir est indispensable il ne doit pas pour autant être clos dans l'enceinte des faits passés qui justifie la culpabilité et s'arrêter au remords et à la quête du pardon. Il devrait, aussi, déclencher un processus d'identification des filiations des faits morbides qui ont fait ces coupables pour mieux conduire la guerre d'éradication de leurs formes nouvelles et inédites.

10. A titre d'exemple pour mettre en évidence ce qui peut servir de base au ressentiment d'un individu ou d'un groupe affronté à l'exclusion :

1 - « Non, la bête immonde, celle qui se revêt de pureté pour refuser l'autre, n'est pas morte. Elle est même parfois tapie derrière les sourires des masques de circonstance. Il y a peu de temps, je me rendais en famille chez une dame, parente éloignée, qui possédait un chien méchant de race show show réputée d'origine chinoise. Etonnée de la placidité de la bête, d'ordinaire agressive, elle s'est écriée en guise de bienvenue:

- Vous avez vu? Il n'a même pas aboyé en vous voyant. Il a reconnu les gens de sa race». Philippe Franchini, Métis, op. cit, p. 128.

2 - «Mais, on l'a dit à plusieurs reprises, cette recherche passionnée d'une culture nationale endeçà de l'ère coloniale tire sa légitimité du souci que se partagent les intellectuels colonisés de prendre du recul par rapport à la culture occidentale dans laquelle ils risquent de s'enliser. Parce qu'ils se rendent compte qu'ils sont en train de perdre, donc d'être perdus pour leur peuple, ces hommes, la rage au cour et le cerveau fou, s'acharnent à reprendre contact avec la sève la plus ancienne, la plus anté -coloniale de leur peuple.»

Frantz Fanon, Les dDamnés de la terre, op. cit., p. 143-144.

11. Ces publications révèlent des enjeux différents relativement aux pratiques venues d'ailleurs dans le temps et dans l'espace : reconnaissance des cultures africaines chez Charles Béart et provinciales avec leurs enjeux didactiques et techniques chez JeanClaude Blanchard, " politique et contestataires » avec le jeu subversif au temps de l'esclavage et à teneur identitaire aujourd'hui à travers le damnyé (de damier : alternance du Noir et du Blanc) chez Gilles Bui-Xuân, éthique chez Martin Buber. Elles entrent dans un corpus au registre commun du point de vue de l'ouverture qui les caractérise à tenir l'autre - celui qui vient d'ailleurs - comme porteur de différences susceptibles de profiter à tous.

12. A titre d'exemple, dans le Bulletin Officiel du Ministère de l'Education Nationale et du Ministère de la Recherche, HS N 6 du 31 Août 2000, Programmes des Enseignements de la classe de seconde générale et technologique, extraits de l'annexe 2 , dans le cadre de l' " Enseignement de détermination ", au niveau de l' "Analyse réflexive » : " (...). Trois compétences méthodologiques de l'analyse réflexive orientent vers des connaissances formulées selon des thèmes d'étude ».

Une de ces compétences peut répondre à la problématique de métissage développée ici : « (...). Porter un regard lucide sur le développement des cultures corporelles » pour laquelle sont sollicitées des " connaissances ", notamment sur le " thème " "Les activités issues d'autres cultures (géographiques ou historiques)». Ce texte des programmes est quelque peu significatif d'une ouverture vers les préoccupations exposées ici. Il a, de ce point de vue, valeur heuristique. Il peut susciter des intérêts pour les différences culturelles à mettre en oeuvre au moyen de la pratique d'activités corporelles inédites relativement 
à l'histoire nationale mais qui gagneraient à être importées volontairement par les enseignants et leurs élèves.

13. Cette manière d'envisager les choses ouvre des possibilités de travail en équipe mettant à contribution les professeurs d'Education Physique, d'Histoire et de Géographie, les documentalistes, les parents d'élèves, les élèves, pour monter des projets de recherches historiques, sociologiques, psychologiques et anthropologiques concernant les grands jeux et les rapports entretenus avec le corps à travers des techniques relevant des cultures d'origine des élèves d'une classe ou d'un groupe de classes donnés. Cela permet une véritable transdisciplinarité motivée par une volonté d'aborder des problèmes concrets rencontrés au quotidien. Faire sauter pour cela les barrières entre universitaires et, par exemple, professeurs du second degré s'avère indispensable pour mener à bien ce type de travail transdisciplinaire et « transstatutaire ». Cette coopération peut être motivée par l'intérêt commun suscité par la nécessité et le bon sens : faire sortir de l'ombre cette duplicité métisse ambiguë et tiraillée tant elle est retranchée dans la clandestinité. Pour ce faire, la mettre en scène et la rejouer acte après acte et en pleine lumière pour donner à ceux qui en subissent les aléas, la chance de se forger un tout identitaire.

\section{RÉSUMÉS}

Les immigrés vivent le métissage au quotidien. A les écouter parler vrai, penser tout haut, agir, le monde irréversiblement voué aux mélanges sera gagnant avec ces pionniers de l'intégration à sens multiple. Celle-ci est encore trop vécue clandestinement tant elle est confrontée à l'intime chez le sujet migrant, à la pression des exclusives culturelles. En effet, malgré le flux important de migrants, c'est toujours la même posture qui est imposée à leurs enfants scolarisés lorsque les éducateurs se contentent de travailler sur une signification étroite et univoque de l'intégration. C'est là mépriser une part importante des valeurs éducatives et culturelles de leurs parents qui peut provoquer du ressentiment chez les intéressés et déboucher sur des violences intériorisées, d'abord contenues, extériorisées ensuite et pour finir parfois explosives. C'est par là même refuser le profit que leurs cultures importées peut apporter aux autochtones de l'Hexagone déjà marqués par la diversité ou, en pensant encore plus écologiquement, à tous les habitants de la Planète.

Pourquoi ne pas prendre plutôt résolument la décision d'actionner la manette des mitigeurs psychologiques, sociaux, éducatifs? La programmation des pratiques en Education Physique et Sportive notamment présente à cet égard des opportunités à saisir.

Immigrants experience interbreeding daily. Listening to their talking straight, thinking at loud and acting, the world, which is irreversibly vowed to mixtures, will come out on top with these pioneers of multiple integration. The latter is still too widely lived clandestinely because it is confronted, on an intimate mode for the migrant individual, with the pressure of cultural exclusiveness. Indeed, despite the considerable influx of migrants, it is still the same position which is imposed on their school-attending children when the teachers content themselves with working on a narrow and univocal meaning of integration. It involves despising a major part of their parents' educational and cultural values, and this can prompt resentment on the part of the 
concerned people and lead to internalised violence, first suppressed, then expressed and eventually sometimes explosive. It amounts to refusing the benefit that their imported cultures can bring to the natives of the Hexagon, who are themselves already marked by diversity, or, following an even more ecological way of thinking, to all the inhabitants of the Planet.

Why not rather resolutely make the decision of activating the levers of psychological, sociological and educational mitigators? The programming of the practices in physical training notably offers in this respect opportunities to be seized.

\section{INDEX}

Mots-clés : éducation physique, métissage, identité

Keywords : violence, intégration, identity, interbreeding, physical training

\section{AUTEUR}

\section{HENRI BOULARAND}

Centre de Recherche sur les Cultures Sportives, Paris XI Orsay. UFR-STAPS-Université Paris 5. 